\title{
Une approche holistique de l'élevage, au cœur des enjeux de santé animale, humaine et environnementale
}

\author{
Michel Duru ${ }^{*}$, Claire Le Bras et Myriam Grillot \\ INRAE, UMR 1248 AGIR, Université Toulouse, INPT, 31326 Castanet Tolosan, France
}

\begin{abstract}
Résumé - Dans les pays occidentaux, les interrogations sur les systèmes d'élevage et la consommation des produits animaux sont de plus en plus nombreuses. Repenser la consommation globale de produits animaux parallèlement à un dimensionnement et une réorientation des élevages devient nécessaire pour des raisons sanitaires et environnementales. Dans ce contexte, nous proposons une approche holistique pour faire émerger des pistes de solutions qui tiennent compte des effets d'interdépendance entre la santé des êtres vivants et de leur environnement et de la diversité des situations. Nous montrons que la réduction de la surconsommation actuelle de produits animaux est nécessaire, en particulier pour la viande. Toutefois, les produits animaux apportent des nutriments d'intérêt pour la santé humaine, et certains systèmes d'élevage agroécologiques contribuent à réduire les impacts ou à fournir des externalités positives. Il s'agit de faire évoluer les modes de consommation tout en reconfigurant et réorientant les systèmes d'élevage. Cela implique, entre autres, une prise en compte des échanges internationaux et une évolution des filières pour différentes productions et races animales ou pour les légumineuses par exemple. Pour finir, nous soulignons l'importance du rôle des politiques publiques pour stimuler la mise en place de ces changements, jusque dans l'assiette du consommateur.
\end{abstract}

Mots clés : biodiversité / changement climatique / maladie chronique / prairie / ruminant

\begin{abstract}
A holistic approach of livestock, at the heart of animal, human and environmental health issues. In Western countries, questions about livestock systems and the consumption of animal products are more and more numerous. Rethinking the overall consumption of animal products in parallel with the sizing and reorientation of farms is becoming necessary for health and environmental reasons. In this context, we propose a holistic approach to bring out possible solutions that take into account the effects of interdependence between the health of living organisms and their environment, and the diversity of situations. We show that the reduction of the current overconsumption of animal products is necessary, in particular for meat. However, animal products provide key nutriments for human health, and some agroecological livestock systems help to reduce impacts or provide positive externalities. It is about changing diets while reconfiguring and reorienting livestock systems. This implies, among other things, taking into account international trade, and changing value chains for animal breeding or legumes, for example. Finally, we stress the importance of the role of public policies in stimulating the implementation of these changes, right down to the consumer's diet.
\end{abstract}

Keywords: biodiversity / chronical disease / climate change / grassland / ruminant

\section{Des enjeux multiples interdépendants, mais des approches partielles}

De nombreuses analyses systémiques récentes concluent à la nécessité de réduire notre consommation de produits animaux dans les pays occidentaux, sur lesquels porte notre étude (Godfray et al., 2018; Springmann et al., 2020; Fresán

\footnotetext{
$\overline{\text { *Auteur de correspondance }}$ : michel.duru@inrae.fr
}

et Sabaté, 2019; Willett et al., 2019). Ces analyses présentent néanmoins des limites thématiques ou méthodologiques qui empêchent d'avoir une vision globale et structurée des changements concomitants à mettre en oeuvre de la production jusqu'à la consommation de produits animaux, ou bien qui rendent difficile la comparaison des résultats.

Une première limite tient à ce que la plupart des travaux ne portent que sur une partie du système alimentaire.

Les études dans le champ de la nutrition et de la santé publique évaluent les impacts des consommations alimentaires 
sur la santé et l'environnement (Perignon et al., 2017), simulent des réductions de la consommation de viande (Vieux et al., 2012), ou cherchent à identifier des choix alimentaires visant à atteindre simultanément des objectifs de santé, d'environnement, d'acceptabilité culturelle et/ou d'accessibilité financière (Barré et al., 2018). Cependant, ces études ne traitent pas, par construction, des conséquences sur la réorganisation des filières d'élevage. Inversement, les politiques publiques européennes se fixent comme objectif d'augmenter fortement l'autonomie protéique de l'élevage sans envisager une réduction de la consommation de viande (Parlement européen, 2018).

Certaines études affichant une vision systémique correspondent en fait à une analyse en silo, ce qui conduit à relativiser les conclusions. Par exemple, Smith et al. (2019) indiquent qu'une extensification de l'élevage du fait du passage en bio en Grande-Bretagne réduirait la sécurité alimentaire. Mais il n'est pas envisagé en parallèle une réduction de la consommation de viande. D'où la nécessité de penser les changements dans les filières animales en cohérence avec des changements de régime alimentaire, et réciproquement. Enfin, s'agissant de prospectives, si de nombreuses études académiques examinent comment réduire les émissions de gaz à effet de serre (GES) (Herrero et al., 2016; Varijakshapanicker et al., 2019), la plupart ne s'assurent pas que leurs propositions permettraient d'atteindre les engagements pris par les pouvoirs publics.

Une limite méthodologique tient à différentes hypothèses quant aux ressources qui sont ou non consommables par l'homme. Ainsi, certaines études portant sur l'efficience protéique (Baber et al., 2018; Laisse et al., 2019) considèrent que les surfaces de maïs ou de colza n'entrent pas en compétition avec l'alimentation humaine, car ces plantes ne sont pas consommées par l'homme. Cependant, ces surfaces pourraient servir à produire des végétaux, des légumineuses par exemple, pour l'alimentation humaine (Mottet et al., 2017). Selon l'hypothèse retenue, l'importance de l'élevage n'entrant pas en compétition avec l'alimentation humaine sera très différente.

Enfin, des études à vocation systémique ne prennent généralement pas suffisamment en compte de manière simultanée, les points critiques suivants:

- la capacité de certains systèmes d'élevage à fournir des externalités positives est souvent ignorée. Pour les ruminants, plusieurs études simulent les effets d'une réduction de la consommation de viande sans considérer les différentes façons de la produire (Westhoek et al., 2014). Ainsi, Schader et al. (2015) proposent de calibrer les effectifs d'animaux en considérant uniquement les prairies permanentes, mais pas les prairies temporaires contenant des légumineuses alors qu'elles fournissent des services écosystémiques (Dumont et al., 2018; Martin et al., 2020).

- dans les pays très consommateurs de protéines animales, les scénarios d'utilisation des terres ne tiennent pas compte de la grande hétérogénéité de consommation de viande, si bien que les réductions possibles sont surestimées du fait que certaines personnes en consomment moins que l'hypothèse moyenne retenue.

- des marges de manœuvre sont possibles grâce aux morceaux de viande actuellement peu valorisés. Les choix préférentiels des consommateurs quant à la nature des



Fig. 1. Modèle pour l'analyse des enjeux de l'élevage et de l'alimentation en termes de santé.

Fig. 1. Framework for analysing livestock and food issues in terms of health.

morceaux de viande consommés (avant, arrière, abats) sont souvent ignorés, alors qu'ils structurent l'organisation des filières et les volumes d'import-export.

- les apports en vitamine B12, calcium et fer justifient de la consommation de produits animaux, mais les études prennent trop peu en compte l'intérêt des apports en acides gras indispensables par les produits animaux et dont la teneur dépend beaucoup du mode d'alimentation des animaux (Duru et Magrini, 2016).

Considérer l'ensemble de ces limites et mobiliser les connaissances analytiques permettant de les instruire pour déterminer la part des produits animaux dans notre alimentation et la place de l'élevage dans les territoires nécessite de s'inscrire dans un cadre d'analyse global (Miller et al., 2020; Weindl et al., 2020). C'est pourquoi nous proposons d'adopter une approche holistique de "santé globale» (Duru et Therond, 2019) pour prendre en compte les impacts de l'élevage et de la consommation de produits animaux aussi bien sur la santé animale, humaine, qu'environnementale.

Sur cette base, nous examinons dans une première partie les impacts des systèmes d'élevage et de la consommation de produits animaux sur: i) la santé environnementale à un niveau global comme le dérèglement climatique, ou local comme la pollution de l'air, des eaux ou du sol; ii) la santé humaine en lien avec la valeur santé des produits; iii) la santé animale en lien avec le bien-être animal. Cette analyse nous permet de mettre en évidence plusieurs pistes de solutions qui incluent une réflexion sur: i) le dimensionnement, le type d'élevage (ruminants et monogastriques) et les conduites d'élevage; ii) la transformation des matières premières et leur mode de distribution; iii) le régime alimentaire. Enfin, nous concluons en présentant plusieurs pistes d'action pour faciliter l'organisation des différents acteurs du système alimentaire pour répondre à ces enjeux (Fig. 1). 


\section{Impacts environnementaux de l'élevage et de la consommation de produits animaux}

\subsection{Santé des écosystèmes et sécurité alimentaire}

\subsubsection{Climat: émissions de gaz à effet de serre et} séquestration de carbone

L'élevage est un fort émetteur de gaz à effet de serre (GES) : entre 630 et $863 \mathrm{MtCO}_{2}$ eq, soit de 12 à $17 \%$ des émissions totales de l'UE-27 en 2007. Ces estimations incluent les émissions associées à la production des aliments pour animaux: céréales, protéagineux, maïs et autres fourrages, herbe, paille et coproduits, domestiques ou délocalisés. Le porc, la viande bovine et le lait contribuent respectivement pour 26,28 et $32 \%$ des émissions ; les volailles pour $11 \%$, et les petits ruminants pour $3 \%$ (Bellarby et al., 2013).

En France, les émissions de méthane $(\mathrm{CH} 4$, fermentation entérique et déjections animales) représentent $47 \%$ des émissions de l'agriculture, celles de protoxyde d'azote $\left(\mathrm{N}_{2} \mathrm{O}\right.$, volatilisation à partir des engrais azotés et des déjections) $43 \%$ et celles de dioxyde de carbone $\left(\mathrm{CO}_{2}\right.$, énergie fossile) $10 \%$ (Ademe, 2011). En considérant des émissions de $\mathrm{CO}_{2}$ et $\mathrm{N}_{2} \mathrm{O}$ au prorata de l'utilisation des cultures par l'élevage (environ $1 / 3$ des surfaces cultivées), mais avec une fertilisation des prairies 3 fois moindre que les cultures, on peut attribuer 20 à $25 \%$ des émissions de $\mathrm{CO}_{2}$ et $\mathrm{N}_{2} \mathrm{O}$ à l'élevage, soit environ $70 \%$ des émissions totales en incluant le méthane. Cette valeur est cohérente avec l'estimation de la contribution aux émissions de GES des produits animaux dans notre alimentation. Ainsi, l'étude NutriNet-Santé a montré que les produits animaux contribuent à $65 \%$ des émissions de GES des diètes individuelles du $5^{\mathrm{e}}$ des personnes consommant le moins de produits animaux, et $80 \%$ pour le $5^{\mathrm{e}}$ en consommant le plus (Lacour et al. 2018); la viande y participant pour $50 \%$ et $65 \%$. Les estimations moyennes de Pointereau et al. (2019) correspondent à la fourchette haute. Ce fort impact de l'élevage et d'un régime alimentaire de type occidental vient du fait que pour $100 \mathrm{~g}$ de protéines, les émissions brutes sont bien supérieures pour la viande rouge (15 à $30 \mathrm{~kg}$ en équivalent $\mathrm{CO}_{2}$ ) que pour les protéines végétales issues de légumineuses $(2-3 \mathrm{~kg})$; les valeurs pour le porc, les volailles, le bœuf issu du troupeau laitier, les laitages (fromages) étant intermédiaires (5 à $15 \mathrm{~kg}$ ) (Poore et Nemecek, 2018). Ces valeurs moyennes élevées sont consubstantielles à l'élevage, mais recouvrent une variabilité (Aleksandrowicz et al., 2016; Dumont et al., 2018).

Pour tenir les engagements de neutralité carbone en 2050, les experts s'accordent à dire qu'il faudra diviser les émissions de GES au minimum par 2 pour l'agriculture (Ministère de la transition écologique et solidaire, 2020). Cette réduction n'a été que de $5 \%$ sur la période $1990-2015$ et devrait être de $46 \%$ d'ici 2050 pour atteindre les objectifs; son rythme doit donc être considérablement accéléré $(\times 7)$. Des estimations montrent un potentiel de réduction des émissions par les bonnes pratiques en agriculture et en élevage de $13 \mathrm{MtCO}_{2}$ eq, dont 5,5 pour l'élevage (Pellerin et al., 2013), soit $15 \%$ environ des émissions du domaine ( $\left.88 \mathrm{MtCO}_{2} \mathrm{eq}\right)$. Elles ne permettront cependant pas de diviser par deux les émissions de GES.

Outre la réduction des émissions, il est possible de séquestrer plus de carbone, notamment par l'élevage de ruminants à l'herbe. Or, les estimations de stockage additionnel par les prairies permanentes varient selon la méthode utilisée (Pellerin et al., 2019) et sont très dépendantes de l'âge des prairies; celles de plus de 50 ans arrivant à un état de saturation en carbone (Smith, 2014). Pour la France, ce stockage additionnel est estimé à $2,7 \mathrm{MtCO}_{2}$ eq pour les prairies (Pellerin et al., 2019). Toutefois, cette valeur est peutêtre sous-estimée compte tenu des incertitudes pour les horizons profonds (Barré et al., 2017). Cette valeur est toutefois plus faible que les émissions de GES de l'agriculture, et ne peut donc les compenser.

L'objectif de neutralité carbone nécessite d'envisager des innovations systémiques, dont une révision de la place de l'élevage dans l'agriculture et de celle des protéines animales dans notre alimentation.

\subsubsection{Biodiversité : contribution à son érosion mais fourniture de services écosystémiques}

Le système alimentaire est un des principaux facteurs engendrant une érosion de la biodiversité avec une destruction d'habitats sources de biodiversité (forêts et prairies) en faveur des cultures. Les pays de l'Europe de l'Ouest sont importateurs nets d'impacts au travers des échanges commerciaux, et ce, principalement auprès des pays tropicaux (Chaudhary et Kastner, 2016). Ainsi, ces cinquante dernières années, face à l'augmentation de la demande en Union européenne, et plus récemment en Chine, la production du soja a décuplé, passant de 27 à 267 millions de tonnes (Lassaletta et al., 2013). En France, les importations de tourteaux de soja sont actuellement d'environ 3 millions de tonnes (Duru, 2019). Cette croissance provient essentiellement de la déforestation et de la conversion des prairies en cultures en Amazonie et au Sud du Brésil (Boerema et al., 2016). En Europe, l'accroissement des importations de soja a été concomitante à la baisse des surfaces de prairies pour des raisons de rentabilité, générant aussi une érosion importante de la biodiversité. En outre, la mise en contact des animaux sauvages chassés de leur habitat naturel avec des élevages domestiques dans des écosystèmes déséquilibrés offre à des agents infectieux de nouvelles chaînes de transmission et des recompositions possibles favorisant l'émergence de maladies infectieuses (Wilkinson et al., 2018).

La croissance rapide de la production de soja et ce, à faible coût pour les pays importateurs, n'a pu se produire que concomitamment au développement de l'élevage intensif (Oliveira et Schneider, 2015). Cela a généré des surplus d'éléments minéraux dans les régions à forte concentration géographique de l'élevage, ne permettant pas un recyclage raisonné.

En Europe, il est montré que l'agriculture est la première source de pollution par les nutriments dans les eaux de surface, entraînant environ $60 \%$ des fuites en azote (dont la moitié dans les zones où il est concentré bien que représentant bien moins de $50 \%$ des surfaces) et environ $30 \%$ des fuites en phosphore vers les milieux aquatiques (Bouraoui et al., 2009). L'élevage contribue aux émissions d'ammoniac (93\% en Europe selon Eurostat, 2013). Or, l'ammoniac se recombine dans l'atmosphère avec des oxydes d'azote et de soufre pour former des particules fines $(\mathrm{PM} 2,5)$ préjudiciables à la santé humaine. Il contribue aussi à l'acidification des milieux terrestres, aquatiques et aériens et indirectement, à l'eutrophisation, 
ainsi qu'aux émissions de GES par nitrification et dénitrification dans les sols. À l'inverse, les élevages de ruminants herbagers (souvent liés à l'agriculture biologique) fournissent des services à la société et à l'agriculture en jouant le rôle d'infrastructure écologique (pollinisation, hébergement d'ennemis naturels des cultures) (Michaud et al., 2021). Les prairies sont une source d'azote lorsqu'elles contiennent des légumineuses (Martin et al., 2020), et contribuent à créer des paysages en mosaïque (Sirami et al., 2019). À l'opposé, les formes d'élevages qui mobilisent un nombre limité de cultures : céréales (blé, orge, maïs) et oléagineux (colza pour les tourteaux), contribuent peu à la biodiversité, les paysages associés étant plus homogènes.

\subsubsection{Sécurité alimentaire: le rôle clef de la nature des protéines}

La sécurité alimentaire est caractérisée en terme de surface nécessaire pour nourrir une personne ou, inversement, en termes de calories ou de protéines produites sur une surface donnée. Il faut de 5 à 10 fois plus de surface pour produire une même quantité de protéines animales que de protéines végétales (Poore et Nemecek, 2018).

Pour évaluer les impacts en termes de sécurité alimentaire, ces données sont à compléter car toutes les ressources utilisées pour nourrir les animaux, comme l'ensilage de maïs, les graines de colza ou la luzerne déshydratée, ne sont pas consommables par l'homme. C'est pourquoi, contrairement au choix fait pour le calcul de l'efficience protéique (rapport entre protéines animales produites et protéines végétales utilisées pour les produire) (Laisse et al., 2019), nous avons considéré les surfaces et non les plantes qui entrent en compétition avec l'alimentation humaine, puisque les surfaces hors prairies permanentes pourraient être utilisées par exemple pour produire des protéines végétales. Sur ces bases, 30\% (AGRESTE, 2013) à 40\% (Duru et al., 2017a, 2017 b) du lait étant produit à l'herbe en France, il reste 60 à $70 \%$ des surfaces qui auraient pu avoir une autre destination. Pour les élevages allaitants, la contribution des prairies est bien plus élevée, environ $80 \%$. Outre les prairies, les ruminants utilisent, sans compter les coproduits, 1,8 millions d'hectares de maïs, et des céréales estimées à 1 million d'hectares sur la base d'un rendement de $60 \mathrm{q} /$ ha pour $6,1 \mathrm{Mt}$ consommées (Cordier et al., 2020), soit $30 \%$ de la surface agricole utile (SAU) hors prairies permanentes. Les coproduits de l'industrie alimentaire n'entrant pas en compétition avec l'alimentation humaine ne dépassent pas, pour les monogastriques, $15 \%$ de la ration (AGRESTE, 2013). Bien que l'ensemble des surfaces hors prairies permanentes ne puisse être mise en culture pour l'alimentation humaine, et que certaines inter-cultures puissent être utilisées pour l'alimentation animale, on peut conclure que l'élevage entre fortement en compétition avec l'alimentation humaine. Cependant, compte tenu des ressources non consommables par l'homme (prairies permanentes, coproduits), l'optimisation de l'utilisation des terres par habitant nécessite une consommation minimale de produits animaux. Il a ainsi été montré aux Pays-Bas que les protéines animales devaient constituer au minimum $12 \%$ des apports protéiques (au lieu de $50 \%$ dans le régime actuel) pour optimiser l'usage des ressources (Van Kernebeek et al., 2016).

\subsection{Santé animale et humaine}

\subsubsection{Bien-être animal: des conditions d'élevage qui peuvent le remettre en cause}

Le bien-être animal concerne quatre domaines (nutrition, environnement, santé, comportement) et est un enjeu pour l'ensemble des systèmes d'élevage (Temple et Manteca, 2020). L'un des enjeux se rapporte à la santé, soit l'absence de maladies liées au fort niveau de production des animaux (troubles locomoteurs, ascites des poulets, mammites et boiteries chez les vaches laitières, etc.). Les autres concernent : i) les pratiques douloureuses (écornage, castration, épointage du bec ou des dents) ; ii) le confinement et la concentration des animaux qui limitent leur déplacement, accentuent les agressions mutuelles, favorisent les maladies ; iii) la pauvreté de leur habitat qui entraîne l'ennui et des comportements anormaux; iv) les manipulations brusques lors des transports et le mauvais étourdissement avant la saignée à l'abattoir. Les atteintes au bien-être causées par de mauvaises conditions de logement peuvent concerner l'ensemble des productions et espèces. Les différences entre élevages sont considérables et l'avantage n'est pas forcément observé pour les élevages bio par rapport aux conventionnels (Van Wagenberg et al., 2017), ni pour les petits relativement aux grands (Robbins et al., 2016).

\subsubsection{Valeur santé des produits animaux: l'alimentation animale et le type de transformation des produits peuvent la dégrader}

Au-delà de leur intérêt gustatif et des traditions gastronomiques, les produits animaux apportent du fer, du calcium et de la vitamine B12. Les protéines végétales sont moins digestibles que les protéines animales (Ertl et al., 2016) et moins équilibrées entre les différents acides aminés. Cependant, cet inconvénient des protéines végétales peut être corrigé en associant la consommation de légumineuses et de céréales. Certains produits animaux, notamment les viandes rouges et les charcuteries, sont considérés comme cancérigènes lorsque consommés en excès (De Smet et Vossen, 2016). Or en France, respectivement $40 \%$ et $70 \%$ de la population consomment plus que les valeurs maximales préconisées par le Plan national nutrition-santé 4 (PNNS 4) : $500 \mathrm{~g}$ de viande rouge et $150 \mathrm{~g}$ de charcuterie par semaine.

L'apport des produits animaux en acides gras essentiels non synthétisés par l'Homme (oméga-3, particulièrement le DHA et l'EPA, et oméga-6) est essentiel pour le bon fonctionnement du système immunitaire et réduit le risque de maladies chroniques. Les oméga- 3 et 6 ont respectivement une fonction anti-inflammatoire et inflammatoire. Or $95 \%$ de la population française présente un déficit prononcé en oméga3, notamment en DHA et EPA, et le rapport moyen oméga-6/ oméga-3 de notre diète est d'environ 10, au lieu de 5 recommandé pour éviter les inflammations chroniques à bas bruit (Simopoulos et Di Nicolantonio, 2016). L'une des raisons de ce déficit en oméga-3 est que l'alimentation animale, tout comme la nôtre, n'est pas toujours optimale. Tous les produits animaux contiennent ces acides gras, mais la plupart ont un rapport oméga-6/oméga-3 supérieur à 5 (Duru, 2019). Ils contribuent au déséquilibre de notre alimentation plus qu'au contrôle des inflammations (Simopoulos et Di Nicolantonio, 2016). Seuls les produits laitiers issus d'une alimentation à 
Tableau 1. Comparaison de différents régimes alimentaires pour les protéines consommées et les émissions de gaz à effet de serre (GES). Table 1. Comparison of different diets for protein intake and greenhouse gas (GHG) emissions.

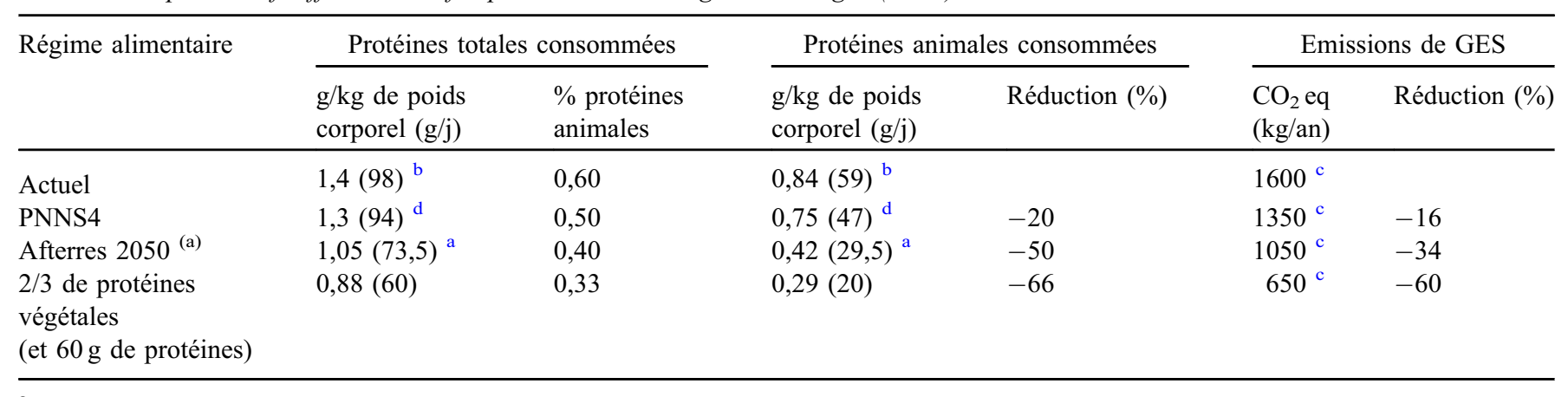

\footnotetext{
a Solagro (2014).

b Étude individuelle nationale sur les consommations alimentaires 2006-2007.

c Pointereau et al. (2019).

${ }^{\mathrm{d}}$ Quantité de protéines animales estimées à partir des recommandations du PNNS4 (g/j) : 70 (viande rouge); 22 (charcuterie), 30 (fromage), 30 (poisson) et des hypothèses suivantes : 45 pour le poulet, supposé constituer $1 / 3$ de la viande (soit environ $47 \mathrm{~g}$ de protéines/jour); les autres caractéristiques ont été calculées sur la base de ces valeurs et de la proportion de protéines animales dans l'assiette ; les émissions de GES ont été estimées au prorata de la quantité de protéines animales.
}

l'herbe ou la viande résultant d'une finition à l'herbe (ou complémentation au lin) fournissent des produits plus riches en oméga-3 et un rapport oméga-6/oméga-3 inférieur à 5 . Pour les viandes de volaille et de porc ainsi que les œufs, la complémentation des animaux avec du lin permet de tripler la teneur de ces produits en oméga-3, y compris le DHA et l'EPA. Elle ne permet cependant pas d'atteindre les recommandations (Duru et Magrini, 2016) et ne touche que 5 à $10 \%$ du marché (Duru, 2019).

\subsubsection{Le rôle du microbiote intestinal dans la santé des animaux et des hommes}

Le rôle pivot du microbiote intestinal dans les fonctions physiologiques de la santé des vertébrés est largement prouvé. L'amélioration de la fonctionnalité gastro-intestinale par l'alimentation est de la plus haute importance compte tenu de ses effets positifs sur la santé et le bien-être des animaux, mais aussi sur les performances de production, en limitant la perte d'efficacité alimentaire et l'utilisation d'antibiotiques (Kraimi et al., 2019).

Pour l'homme, le déséquilibre entre types de protéines et d'acides gras (typique du régime alimentaire occidental) est source de maladies chroniques inflammatoires, obésité et diabète, notamment $\mathrm{du}$ fait d'une dysbiose intestinale provenant de la réduction de la diversité du microbiote (Rinninella et al., 2019). Les facteurs les plus connus en sont un déficit en fibres et en oméga-3, mais aussi un excès de produits sucrés, salés, ultra-transformés (Shi, 2019), et d'une trop grande utilisation d'antibiotiques; l'excès de consommation d'acides gras saturés faisant débat.

\subsubsection{La surconsommation de protéines animales n'est bonne ni pour la santé humaine, ni pour celle des écosystèmes}

En France, la consommation de protéines $(1,4 \mathrm{~g} / \mathrm{kg}$ de poids corporel et par jour) est bien supérieure aux besoins $(0,8)$ (AFSSA, 2007) et aux recommandations (1 à $1,2 \mathrm{~g} / \mathrm{kg}$ de poids corporel) (Rémond, 2016). La proportion de protéines animales (environ $2 / 3$ des apports) devrait être ramenée à $50 \%$ (PNNS 4). Sur la base de cette recommandation, et en limitant un peu la consommation totale de protéines, on trouve une réduction de la consommation de viande d'environ $20 \%$ et des émissions de GES de 16\% (Tab. 1). Ainsi, adopter un régime de type méditerranéen, comme défini par Dernini et al. (2017), basé sur les recommandations optimales en protéines, entre 1 et $1,2 \mathrm{~g} / \mathrm{kg}$ poids corporel/jour, avec équilibre entre protéines végétales et animales, proche de celui considéré dans le scénario Afterres 2050 (Solagro, 2014), réduirait la consommation de protéines animales de $50 \%$ et les émissions de GES d'environ $34 \%$ (Pointereau et al., 2019). S'aligner sur le strict besoin en protéines avec $2 / 3$ de protéines végétales permettrait de réduire les émissions de GES de $60 \%$ (Tab. 1).

Bien que la comparaison soit difficile à faire, ces résultats sont cohérents avec ceux estimés par deux méthodes différentes.

D’une part, Barré et al. (2018) ont montré par une modélisation quantitative qu'une réduction des impacts environnementaux de 30\% (émissions de GES, mais aussi eutrophisation et acidification) est possible en réduisant la consommation de viande d'environ $73 \%$ et $66 \%$ selon qu'il est ou non tenu compte de la biodisponibilité des nutriments, tout en satisfaisant aux apports nutritionnels recommandés. Ces valeurs sont dans la fourchette de celles trouvées pour les scénarios Afterres et celui correspondant à $2 / 3$ de protéines végétales. Les réductions sont respectivement de $-50 \%$ et $-66 \%$ de consommation de protéines animales, soit un peu plus en viande si on considère que la réduction des produits laitiers est plus faible.

D'autre part, Lacour et al. (2018) ont estimé les émissions de GES à partir de l'enquête NutriNet-Santé. Elles varient de 2,8 à $5,4 \mathrm{~kg} \mathrm{CO}_{2}$ eq pour les cinquièmes de l'échantillon consommant le plus $(165 \mathrm{~g} / \mathrm{j})$ et le moins $(72 \mathrm{~g} / \mathrm{j})$ de viande pour une énergie ingérée proche allant de 1900 à $2220 \mathrm{kcal} / \mathrm{j}$. En considérant les recommandations du PNNS 4 (ne pas dépasser la consommation de $500 \mathrm{~g}$ de viande rouge et $150 \mathrm{~g}$ de charcuterie par semaine et en considérant que le porc est 
consommé aux 3/4 sous forme de charcuterie), les quintiles 2 et 4 correspondent respectivement aux recommandations pour la viande rouge et les charcuteries. À partir de ces données, il est possible de calculer la réduction moyenne des émissions : $0,29 \mathrm{~kg} \mathrm{CO}$ eq/j (quintile 2) et $2,04 \mathrm{~kg} \mathrm{CO}_{2} \mathrm{eq} / \mathrm{j}$ (quintile 4), soit 7 à $50 \%$ des émissions moyennes. Ces estimations encadrent la valeur que nous avons indiquée dans le tableau 1. Les estimations faites à partir de NutriNet-Santé en prenant comme référence les recommandations de la commission EATLancet montrent une réduction possible de $56 \%$ des émissions de gaz à effet de serre (Kesse-Guyot et al., 2021). Ces résultats sont vraisemblablement sous-estimés puisque les participants à NutriNet-Santé consomment environ $10 \%$ de moins de produits animaux qu'un échantillon représentatif de la population (Andreeva et al., 2016).

L'ensemble de ces études basées sur des méthodes différentes convergent vers une forte réduction de la consommation de protéines pour des raisons environnementales, sans que celle-ci porte préjudice à la santé. Il convient aussi de noter que les réductions d'émissions de $30 \%$ (soit environ $30 \mathrm{MtCO}_{2}$ eq) sont supérieures à celles permises par les bonnes pratiques de culture et d'élevage ( $c f$. Sect. 2.1.1). Diviser par deux les émissions de GES suppose donc de cumuler un régime de type méditerranéen ( $c f$. Afterre dans le Tab. 1) ainsi que les bonnes pratiques (15\%), ce qui au total permettrait une baisse de $49 \%$ des émissions de GES. Un ajustement modéré de l'alimentation ne permettrait donc pas d'atteindre l'objectif affiché dans les politiques publiques.

\subsubsection{L'excès d'utilisation d'antibiotiques, cause d'antibiorésistance}

L'émergence de bactéries multi-résistantes aux antibiotiques a été favorisée par l'usage excessif d'antibiotiques en médecine vétérinaire et humaine. En effet, les bactéries exposées aux antibiotiques évoluent et développent des mécanismes de défense qui leur permettent d'échapper à leur action, un phénomène touchant aussi bien les bactéries à l'origine des infections (bactéries pathogènes) que celles généralement inoffensives qui sont naturellement présentes sur notre corps (bactéries dites commensales), chez les animaux et dans l'environnement. Une fois l'antibiorésistance développée chez l'une ou l'autre de ces espèces bactériennes, elle peut être transmise à d'autres espèces et contribuer à l'expansion du phénomène et à sa diffusion. Les antibiotiques deviennent alors inefficaces (Koch et al., 2017).

En France la consommation d'antibiotiques en médecine vétérinaire est de $530 \mathrm{t}$ /an et est inférieure à la médecine humaine (756t/an). Le plan Antibio a permis une réduction de près de $30 \%$ ces dernières années. Les consommations d'antibiotiques par animal sont très différentes entre les pays européens; la France se situe dans la fourchette moyenne-haute, des marges de progrès existent donc encore pour certains systèmes d'élevage en France (Santé publique France, 2018).

\section{Quelle consommation de produits animaux, et quels niveaux et formes d'élevage?}

Etant donné les interdépendances entre enjeux, les changements à opérer doivent être systémiques. Ils doivent s'appuyer sur les situations identifiées comme les meilleures pour la santé humaine, la santé animale et la qualité des produits, ainsi que la santé des écosystèmes, tout en s'inscrivant dans l'impératif de réduction des émissions de GES. Une représentation schématique des changements à opérer en termes de surfaces, volumes et modalités est présentée sur la figure 2.

\subsection{La consommation de produits animaux doit diminuer et s'orienter vers des produits à haute valeur nutritionnelle}

\subsubsection{Jusqu'où végétaliser l'assiette?}

Pour être plus durable, répondre aux engagements de neutralité carbone en 2050, mais aussi pour assurer notre bon état de santé (Tab. 1), l'alimentation doit avant tout être plus végétale dans les pays occidentaux. Une méta-analyse $(n=63)$ à l'échelle mondiale montre que le passage du régime de référence à des régimes méditerranéen, végétarien et vegan réduirait, selon le pays, les émissions de GES de $10 \%, 32 \%$ et $46 \%$ (Aleksandrowicz et al., 2016; Kim et al., 2020). Ces recommandations sont confortées par des modélisations quantitatives à l'échelle du monde (Mora et al., 2020). Cette réduction des protéines animales ne doit néanmoins pas se faire au profit de n'importe quelles protéines végétales. Les substituts végétaux de viande sont souvent élaborés avec des émulsifiants et édulcorants contre-indiqués pour la santé (Mozaffarian, 2020). La viande in vitro présente aussi des limites pour fournir tous les atouts de la viande en terme de diversité (Chriki et Hocquette, 2020) et il sera nécessaire de réaliser des évaluations complémentaires des coûts et bénéfices de ces technologies qui soient indépendantes des entreprises qui fabriquent ces produits (Santo et al., 2020). À l'inverse, la consommation accrue de protéines végétales, notamment grâce aux légumineuses, permet d'apporter, outre les protéines, des fibres, ainsi que des micronutriments d'intérêt (Champ, 2018). Par ailleurs, les services que les légumineuses fournissent à l'agriculture, par exemple en termes d'émissions moindres de GES, ne sont pas négligeables.

\subsubsection{Les avantages d'accroître l'offre en produits à haute valeur nutritionnelle et valorisant l'animal entier}

La réduction de la production et de la consommation des protéines animales doit en même temps être orientée en termes de composition des produits, de types et d'origine des produits, et ce, pour éviter des effets rebonds en termes de santé, d'environnement et de marché.

Il importe de privilégier des produits animaux dont la teneur est la plus élevée en nutriments dont nous sommes fortement déficitaires. Hormis l'élevage tout à l'herbe ou certaines productions sous cahiers des charges telle que la marque Bleu Blanc Coeur (monogastriques et ruminants), les conduites d'élevage actuels ne maximisent pas la teneur en acides gras essentiels dans les produits animaux (Duru, 2019). Élaborer et mettre en marché de tels produits est nécessaire dans un contexte de réduction de la consommation de viande afin de ne pas aggraver davantage les déficiences nutritionnelles constatées. De plus, le choix des types de viande consommés n'est neutre ni pour la santé ni pour l'agriculture. La prédilection des consommateurs et de la restauration pour 


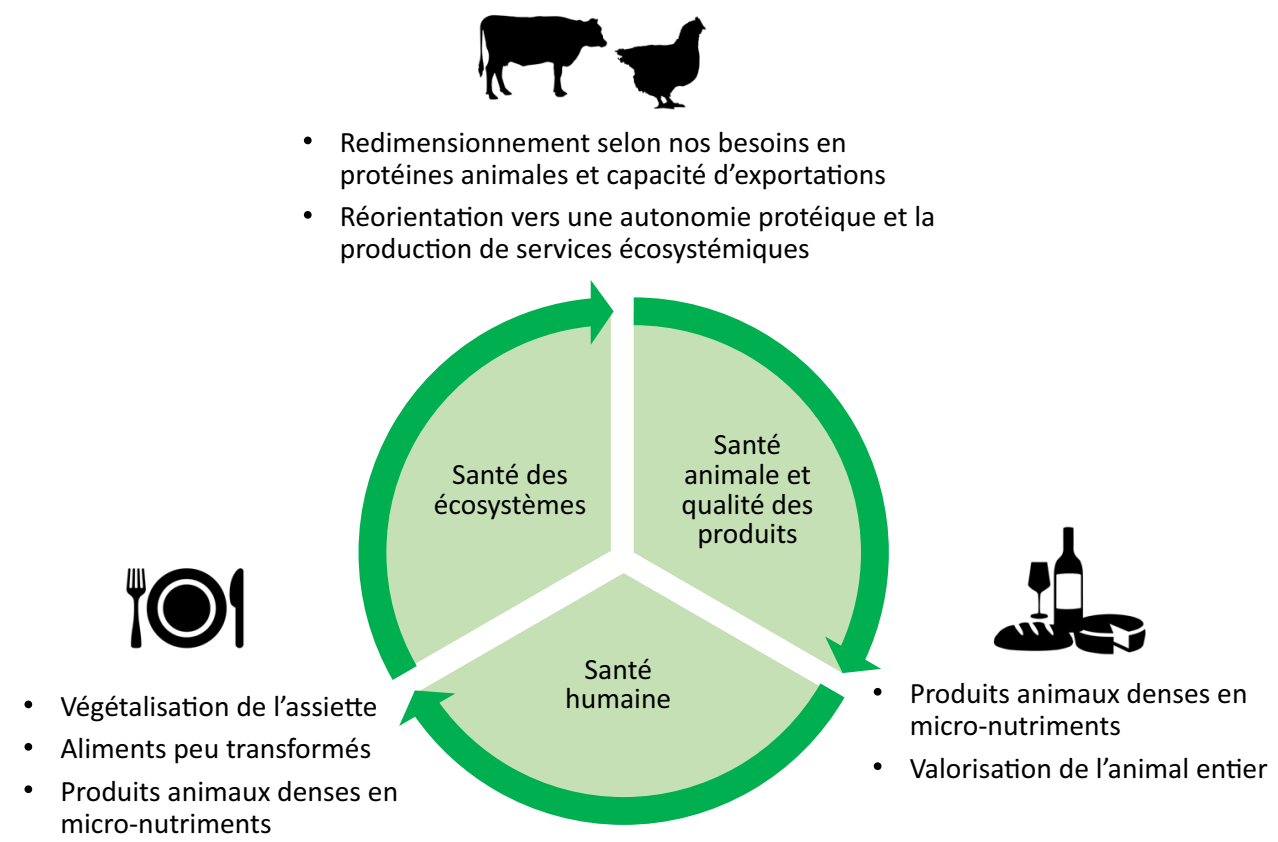

Fig. 2. Changements nécessaires pour une transition vers un système alimentaire plus sain et plus durable: redimensionner l'élevage, les importations et exportations pour atteindre des objectifs environnementaux (diviser par deux les émissions de gaz à effet de serre), assurer un meilleur bien-être animal et une meilleure santé humaine.

Fig. 2. Changes needed for a transition to a more healthy and sustainable food system: resizing livestock production, imports and exports to achieve environmental objectives (halve greenhouse gas emissions), ensure better animal welfare and human health.

certaines pièces de viande (blanc de poulet, steak haché pour burger) au détriment des abats ou bas-morceaux, oriente profondément les filières en Europe, mais aussi les déstructure dans les pays en développement. La consommation des abats, plus riches en micronutriments que les pièces de viande, en vitamine B12 par exemple, devrait être encouragée à la fois pour des raisons de santé et pour éviter le gaspillage.

Au niveau de la transformation, il importe de concevoir des produits transformés sains valorisant et rendant attractifs tous les morceaux de viande (bonne valeur nutritionnelle, goût, traçabilité) pour encourager les consommateurs et la restauration collective à les utiliser. Cette option est particulièrement importante pour les abats, insuffisamment consommés depuis la crise de la vache folle.

\subsection{L'élevage doit être redimensionné et réorienté pour la santé environnementale, humaine et animale}

\subsubsection{Des formes d'élevages agroécologiques à encourager}

Les systèmes d'élevage doivent évoluer en s'inscrivant dans une démarche de transition agroécologique qui valorise de multiples processus écologiques ainsi que les potentialités des territoires.

Pour les ruminants, l'élevage herbager devrait être le système dominant, étant donné sa supériorité en termes d'externalités positives tant pour l'environnement que pour la santé. Bien que les systèmes herbagers ou en bio aient des performances par animal en moyenne $15 \%$ inférieures aux systèmes conventionnels (Gaudaré et al., 2021), ils rendent plus de services à la société que les systèmes plus intensifs
(Duru et Therond, soumis). En système bovin laitier, une alimentation à l'herbe permet d'être gagnant dans les trois domaines - environnement, bien-être animal et santé humaine-, tout en générant des revenus plutôt supérieurs aux systèmes courants (Civam, 2018). Cependant, en France, le lait fait à l'herbe ne représente qu'environ respectivement 4 et $35 \%$ du lait produit en bio et en conventionnel.

Pour la viande issue des troupeaux allaitants, être gagnant dans les trois domaines suppose un engraissement à l'herbe, difficile voire impossible pour les races de gros gabarit courantes en Europe. Ainsi, si les élevages allaitants sont majoritairement basés sur les prairies, l'engraissement à l'herbe, nécessaire pour conserver ses atouts santé (teneur en oméga-3 et en certains micronutriments), a été évalué à moins de $25 \%$ de la viande produite (Duru et al., 2017b). Pour développer l'engraissement à l'herbe, il faudrait privilégier des animaux de plus petit gabarit, par exemple issus du croisement avec des animaux de race Angus, ou des races allaitantes de plus petit gabarit (Vazeille et al., 2018). Ces produits seraient, en plus, mieux adaptés à l'orientation du marché (petites pièces de viande), tout en permettant une diminution de l'impact environnemental, puisque lait et viande se répartissent les émissions de GES. Une supplémentation en lin garantit un engraissement à bonne teneur en oméga-3.

L'entretien des zones difficiles d'accès ou à faible productivité (parcours, friches) devrait être assuré par des petits ruminants, en veillant à disposer de races permettant de mieux valoriser ces milieux (Napoléone et al., 2019). Une analyse comparative de cinq systèmes ovins viande a permis de montrer qu'indépendamment de leur productivité, les systèmes 
saisonnés qui valorisent le mieux l'herbe offrent les meilleures performances économiques et environnementales (Benoit et al., 2019).

Pour les monogastriques, la complémentation en lin pour la valeur santé des produits et l'utilisation de protéines issues de légumineuses produites dans le territoire sont à privilégier pour éviter la déforestation, protéger la biodiversité et réduire les GES (Stinner et al., 2018). Consommer de tels produits permettrait aussi de se rapprocher des apports nutritionnels recommandés en acides gras essentiels (Duru, 2019). Ces pratiques permettent d'éviter les inconvénients du soja importé et ceux du tourteau de colza, dont la culture nécessite beaucoup d'azote et de pesticides. La richesse de ce dernier en oméga-3 n'est cependant pas à négliger. Longtemps légitimé par son rôle de coproduit des agrocarburants, il est de plus en plus remis en cause dans les politiques européennes. L'autonomie en protéines pour les animaux doit donc plutôt être recherchée par le recours à des légumineuses à graines (lupin, féverole) et par une meilleure valorisation des coproduits de l'agroalimentaire ou des déchets de la restauration, ce qui pose des questions de sûreté alimentaire et demanderait un assouplissement de la législation européenne ( $\mathrm{Zu}$ Ermgassen et al., 2016).

Enfin, des systèmes de polyculture-élevage à l'échelle de la ferme ou d'un territoire permettent de fournir une diversité de services si l'intégration entre les deux activités est poussée (Moraine et al., 2016). Volailles et ovins pourraient devenir un coproduit de l'agriculture par exemple en faisant pâturer des intercultures entre deux céréales ou en inter-rang de plantes pérennes. La viande deviendrait ainsi elle-même un coproduit réduisant fortement son empreinte environnementale, valorisant des surfaces et fournissant des services écologiques aux cultures, par exemple par la réduction des risques d'érosion, la création d'habitats pour les ennemis naturels des cultures, etc... Cependant, la gestion fine des interactions entre culture et élevage est plus difficile dans les grandes exploitations. Enfin, dans les territoires à forte concentration animale, les bonnes pratiques d'élevage ne permettent pas de réduire suffisamment les impacts environnementaux, ni de faire bénéficier les cultures des atouts des prairies et des déjections animales (Casal et al., 2019).

\subsubsection{Les échanges internationaux et le dimensionnement des filières doivent être reconsidérés}

En France, adapter l'offre à la demande passe par un dimensionnement à la baisse de la production, en particulier dans les régions à forte densité animale, et/ou par une augmentation des exportations ou une réduction des importations. À ce jour, d'après France Agrimer, la France est exportatrice de produits laitiers (lait: 684 millions de tonnes/an, yaourt : $300 \mathrm{Mt}$, fromage : $310 \mathrm{Mt}$, soit entre 15 et $25 \%$ de la production), de viande de porc ( $8 \mathrm{Mt}$, soit $12 \%$ de la production), mais déficitaire en viande rouge bovine $(-90 \mathrm{Mt}$, soit $6 \%$ de la consommation), en viande ovine $(-80 \mathrm{Mt}$, soit $50 \%$ de la consommation) et en volaille $(-120 \mathrm{Mt}$, soit $6 \%$ de la consommation). Il existe donc des marges de manœuvre pour moduler le seuil d'import-export. Augmenter les exportations des produits à forte typicité (fromage) ou d'intérêt nutritionnel (produits à haute teneur en acides gras indispensables) vers les pays qui n'en disposent pas suffisamment constitue une piste à ne pas négliger pour soutenir les filières. Moins recourir aux importations, du moins pour des produits de standard inférieur à ceux produits en France, est une seconde voie. Cependant ces deux leviers ne peuvent à eux seuls suffire à une baisse de consommation permettant de ne pas dépasser le budget carbone prévu en 2050 et il faut accepter le principe d'un redimensionnement de l'activité.

Les filières d'élevage peuvent anticiper la transition de notre alimentation en se réorganisant à la fois pour produire moins et mieux, avec l'objectif de renforcer leur apport à la santé des écosystèmes, animale et humaine. À cette fin, la nécessaire montée en gamme des produits suppose une meilleure valorisation économique, expliquée au consommateur comme pouvant compenser la réduction conseillée de consommation de viande. Ces changements concernent surtout les élevages intensifs situés dans les territoires à haute densité animale où les impacts environnementaux locaux, eutrophisation et acidification, constituent une limite importante de ces systèmes et ce, malgré des progrès significatifs pour réduire leurs impacts (Dourmad et al., 2017). Ces évolutions supposent donc une réflexion sur la répartition géographique des filières, leur seuil d'import-export et l'organisation de la distribution, afin de répondre aux besoins locaux et de garantir l'autosuffisance alimentaire à différentes échelles. En effet, la spécialisation géographique des territoires d'élevage n'est un avantage que sur le plan économique, la proximité des acteurs dans une même activité permettant de développer des services d'appui, des synergies, des économies d'échelle. Les industries de grande dimension nées dans les régions spécialisées en élevage ont donné à la filière une compétitivité qui a permis d'accroître la taille les élevages, d'où une spirale qui s'autoentretient.

\section{Perspectives: quelle gouvernance de la transition?}

Une analyse de l'élevage et de l'alimentation au prisme de la « santé globale » a permis d'expliciter ces interdépendances. Au-delà d'une recherche de réduction des impacts de l'élevage pour l'environnement et de la consommation de produits animaux pour la santé, il s'agit de promouvoir une diversité de services. Réduire la consommation de produits animaux des consommateurs dépassant les recommandations, redimensionner l'élevage en développant les formes d'élevage d'intérêt pour la santé tout en minimisant l'empreinte environnementale permet d'être gagnant dans les quatre domaines identifiés: environnement local et global, bien-être et santé animale, santé humaine.

Mais concevoir des politiques et stratégies cohérentes entre l'agriculture et l'alimentation pour une alimentation saine et durable est complexe car les enjeux sont multiples, interdépendants, locaux et globaux. Si certains objectifs comme les émissions de GES sont chiffrés, la plupart des autres ne le sont pas, rendant difficile l'évaluation de la cohérence entre les objectifs et les actions. Il est important d'identifier les changements qui permettent des synergies et de traiter plusieurs enjeux en même temps.

Le développement des formes d'élevage agroécologiques décrites en section 3.2 participeraient à une meilleure 
répartition de l'élevage au sein du territoire français, un changement incontournable pour limiter les pertes d'azote qui contribuent aux émissions de GES, à la pollution des eaux et pour réduire l'utilisation de phosphore fossile (Billen et al., 2018). La valorisation des déjections par méthanisation apparaît comme un moyen très efficace de récupérer de l'énergie sans pour autant trop réduire l'apport de fertilisants au sol via les digestats (Laboubee et al., 2020). Tant pour l'agriculture que pour l'alimentation, l'enjeu est donc le changement d'échelle. Compte tenu des interdépendances entre activités, les différents acteurs doivent se coordonner pour engager et soutenir de profondes transformations. Toutefois, leurs intérêts ne sont pas les mêmes, ce qui constitue des freins au changement (Oliver et al., 2018).

Les changements nécessaires à une transition agricole et alimentaire engagent les politiques publiques ainsi que les acteurs des filières tant à l'amont (sélection, agrofourniture) qu'à l'aval de la production (collecte, transformation, distribution) et ce, jusqu'aux consommateurs.

Les acteurs des filières ont un rôle majeur à jouer dans le redimensionnement de l'élevage et de la reconfiguration de leur répartition dans les territoires. Toutefois, les changements doivent s'opérer avec une vision systémique. (Ré-)introduire des élevages dans des zones de grandes cultures peut augmenter une offre déjà excédentaire. Viser l'autonomie protéique des élevages dans leur format actuel éviterait la déforestation importée sans résoudre le problème du surdimensionnement. Redimensionner implique de la reconversion et du renoncement.

Les politiques publiques ont vocation à structurer la cohérence d'ensemble et à stimuler l'engagement des acteurs. Elles peuvent passer par l'établissement de normes : plafonds de chargement (Weishaupt et al., 2020), obligations de moyens (un jour sans viande en restauration collective), recommandations nutritionnelles (PNNS 4), élaboration d'un cadre d'action (projet alimentaire territorial), ou communication et valorisation des initiatives entreprises. Au niveau européen, des dispositifs tels que les «Eco-Schèmes» envisagés dans la Politique agricole commune (PAC) devraient encourager et soutenir des actions qui allient la réduction des émissions territoriales et le stockage de carbone dans les sols avec de nombreux co-bénéfices pour la biodiversité, la lutte contre la déforestation importée, l'emploi ou encore la souveraineté alimentaire. L'arrêt du retournement des prairies et du drainage des zones humides devraient être impératifs, de même que le développement des légumineuses. Enfin, l'expérimentation de l'affichage environnemental, au sein d'une même famille de produits animaux en complément des évaluations nutritionnelles, permettrait de faire des choix plus éclairés par rapport à l'environnement. On pourrait imaginer des effets d'entraînement, de sorte que les entreprises modifient la formulation de leurs produits suivant ces objectifs, en veillant toutefois à ce que ces changements ne s'apparentent pas à du greenwashing (Thorlakson et al., 2018).

La nécessaire réduction des volumes produits et/ou importés pour la consommation intérieure, ainsi que la réorientation des façons de produire nécessitent des accompagnements conséquents des éleveurs par la PAC et la politique agricole française. En outre, ces transitions sont à régionaliser en tenant compte des spécificités des territoires (Dumont et al.,
2016) pour définir localement l'effort de reconception, sans perdre de vue les objectifs de santé et d'environnement, notamment la réduction des émissions de GES fixée au niveau national. Ces efforts de reconversion sont d'autant plus urgents à mettre en œuvre que la place de l'élevage dans notre système alimentaire est remise en cause, notamment concurrencée par d'autres sources de protéines (insectes, culture de cellules). Pour autant, l'élevage n'est pas totalement substituable (services permis par l'élevage), et le remplacement du soja par des insectes n'est pas forcément meilleur pour l'environnement (Thévenot et al., 2018).

Avec la mise en œuvre des mesures de la loi Egalim, de nombreux changements sont en cours dans les secteurs de la restauration collective au niveau national, se répercutant localement. Cette loi oblige tous les responsables de sites de préparation de repas ou prestataires à élaborer des menus végétariens au moins une fois par semaine en tant qu'alternative, mais aussi à introduire des produits sous signe d'identification d'origine et de la qualité, équitables ou encore issus d'un projet alimentaire territorial. Au-delà des $50 \%$ du volume d'achat sous signe d'identification de la qualité et de l'origine, dont $20 \%$ de produits issus de l'agriculture biologique, viser l'introduction de produits permettant de fournir d'autant plus d'externalités positives serait un levier de changement des pratiques des producteurs et des coopératives agricoles environnantes. Compte tenu des volumes demandés par la restauration, cela permettrait d'impulser la structuration des filières locales concernées. Pour les acheteurs publics, cela nécessite une bonne connaissance de l'offre locale et des contraintes des producteurs locaux, une révision des critères de sélection et de jugement des offres de leurs fournisseurs, ainsi qu'une révision de leur plan alimentaire. L'implication des équipes de cuisine, notamment au travers de la formation des agents et leur sensibilisation à l'enjeu de la diversification des protéines, est en parallèle nécessaire. Cette loi à elle seule ne peut être la source de tous les changements, c'est pourquoi des initiatives complémentaires, comme la labellisation des cuisines (Ecocert, 2021), peuvent participer à la transition.

Chacun, en tant que consommateur et citoyen, doit avoir le choix de renforcer aussi bien sa santé individuelle que celle de son environnement. Mais choisir suppose une information facilement accessible quant aux impacts environnementaux et de santé de l'agriculture et de l'alimentation. Les politiques publiques en silo, les informations fragmentées par domaine (agriculture, alimentation), par problème (environnement, santé) ou à différents niveaux (la parcelle, l'animal, la ferme ou le territoire en agriculture, le produit ou le régime alimentaire pour l'alimentation) constituent des freins à une perception équilibrée pour le consommateur et à la prise de décision éclairée par les acteurs économiques. En outre, la sensibilisation des consommateurs est entravée par les croyances sur la nourriture: la nutrition, la santé et le goût sont à la fois des catalyseurs et des obstacles à la volonté, comme le montrent dans une étude récente Sanchez-Sabate et al. (2019).

Pour ce qui est de l'élevage et des protéines, ces difficultés sont particulièrement aiguës compte tenu des interactions multiples entre domaines et niveaux d'analyse, et par le fait que toutes les formes d'élevage ne se valent pas en termes d'impacts sur l'environnement, et n'ont pas les mêmes atouts pour rendre des services à la société. 
À tous les niveaux, le cadre d'analyse proposé se garde de proposer une seule solution "clé en main», car les transitions à opérer doivent tenir compte des fortes différences de consommation de produits animaux, ainsi que des spécialisations agricoles très variables d'une région à l'autre.

Remerciements. Cette recherche a été soutenue par le programme PSDR 4 (Projet ATA-RI; 2016-2020) financé par l'INRAE et la Région Occitanie.

\section{Références}

Ademe. 2011. https://www.ademe.fr/sites/default/files/assets/docu ments/guide-realisation-bilans-emissions-ges-secteur-agricole7403.pdf.

AFSSA. 2007. Apports en protéines : consommation, qualité, besoins et recommandations. http://www.afssa.fr/Documents/NUT-Ra-Pro teines.pdf.

AGRESTE. 2013. L'alimentation animale, principales destinations des productions végétales. Agreste synthèse 2013/208, 9 p.

Aleksandrowicz L, Green R, Joy EJM, Smith P, Haines A. 2016. The impacts of dietary change on greenhouse gas emissions, land use, water use, and health: A systematic review. PLoS ONE 11(11): 116. https://doi.org/10.1371/journal.pone.0165797.

Andreeva VA, Deschamps V, Salanave B, Castetbon K, Verdot C, Kesse-Guyot E, et al. 2016. Comparison of dietary intakes between a large online cohort study (Étude NutriNet-Santé) and a nationally representative cross-sectional study (Étude Nationale Nutrition Santé) in France: addressing the issue of generalizability in eepidemiology. American Journal of Epidemiology 184(9): 660669. https://doi.org/10.1093/aje/kww016.

Baber JR, Sawyer JE, Wickersham TA. 2018. Estimation of humanedible protein conversion efficiency, net protein contribution, and enteric methane production from beef production in the United States. Translational Animal Science 2(4): 439-450. https://doi. org/10.1093/tas/txy086.

Barré P, Angers D, Basile-Doelsch I, Bispo A, Cécillon L, Chenu C, et al. 2017. Ideas and perspectives: Can we use the soil carbon saturation deficit to quantitatively assess the soil carbon storage potential, or should we explore other strategies? pre-print: https:// doi.org/10.5194/bg-2017-395.

Barré T, Perignon M, Gazan R, Vieux F, Micard V, Amiot MJ, et al. 2018. Integrating nutrient bioavailability and co-production links when identifying sustainable diets: how low should we reduce meat consumption? PLOS One 13(2): e0191767. https://doi.org/ 10.1371/journal.pone.0191767.

Bellarby J, Tirado R, Leip A, Weiss F, Lesschen JP, Smith P. 2013. Livestock greenhouse gas emissions and mitigation potential in Europe. Global Change Biology 19(1): 3-18. https://doi.org/ 10.1111/j.1365-2486.2012.02786.x.

Benoit M, Sabatier R, Lasseur J, Creighton P, Dumont B. 2019. Optimising economic and environmental performances of sheepmeat farms does not fully fit with the meat industry demands. Agronomy for Sustainable Development 39(4): 1-11. https://doi. org/10.1007/s13593-019-0588-9.

Billen G, Le Noë J, Garnier J. 2018. Two contrasted future scenarios for the French agro-food system. The Science of the Total Environment 637-638: 695-705. https://doi.org/10.1016/j.scito tenv.2018.05.043.

Boerema A, Peeters A, Swolfs S, Vandevenne F, Jacobs S, Staes J, et al. 2016. Soybean trade: Balancing environmental and socio-economic impacts of an intercontinental market. PLoS ONE 11(5): 1-13. https://doi.org/10.1371/journal.pone.0155222.
Bouraoui F, Grizzetti B, Aloe A. 2009. Nutrient discharge from rivers to seas for year 2000. European Commission, Joint Research Centre, Institute for Environment and Sustainability, 72 p. https:// doi.org/10.2788/38971.

Casal L, Durand P, Akkal-Corfini N, Benhamou C, Laurent F, Salmon-Monviola J, et al. 2019. Reduction of stream nitrate concentrations by land management in contrasted landscapes. Nutrient Cycling in Agroecosystems 114(1): 1-17. https://doi.org/ 10.1007/s10705-019-09985-0.

Champ M. 2018. Devrions-nous manger plus de céréales complètes ? Cahiers de Nutrition et de Diététique 53(1): 22-33. https://doi.org/ 10.1016/j.cnd.2017.12.001.

Chaudhary A, Kastner T. 2016. Land use biodiversity impacts embodied in international food trade. Global Environmental Change 38: 195204. https://doi.org/10.1016/j.gloenvcha.2016.03.013.

Chriki S, Hocquette JF. 2020. The myth of cultured meat: a review. Frontiers in Nutrition 7: 7. https://doi.org/10.3389/fnut.2020.00007.

Civam. 2018. http://www.agriculture-durable.org/wp-content/ uploads/2019/09/Obstekeco-Civam_publi2019_web.pdf.

Cordier C, Sailley M, Courtonne JY, Duglot B, Cadudal F, Perrot C, et al. 2020. Analyse des flux de matières premières en alimentation animale en France. Document édité par le GIS Avenir Elevages (France), $6 \mathrm{p}$.

De Smet S, Vossen E. 2016. Meat: The balance between nutrition and health. A review. Meat Science 120: 145-156. https://doi.org/ 10.1016/j.meatsci.2016.04.008.

Dernini S, Berry EM, Serra-Majem L, La Vecchia C, Capone R, Medina FX. 2017. Med Diet 4.0: The Mediterranean diet with four sustainable benefits. Public Health Nutrition 20(7): 1322-1330. https://doi.org/10.1017/S1368980016003177.

Dourmad JY, Delaby L, Boixadera JL, Ortis C, Méda B, Gaigné C, et al. 2017. Diversité des services rendus par les territoires à forte densité d'élevages, trois cas d'étude en Europe. INRAE Productions Animales 30(4): 303-320. https://doi.org/10.20870/pro ductions-animales.2017.30.4.2261.

Dumont B, Dupraz P, Aubin J, Benoit M, Chatellier V, BouamraMechemache Z, et al. 2016. Rôles, impacts et services issus des élevages en Europe. Synthèse de l'expertise scientifique collective, INRA (France), $133 \mathrm{p}$.

Dumont B, Groot JCJ, Tichit M. 2018. Make ruminants green again how can sustainable intensification and agroecology converge for a better future? Animal 12(s2): s210-s219. https://doi.org/10.1017/ S1751731118001350.

Duru M. 2019. Agri-food choices for health: the case of fatty acids. OCL-Oleagineux Corps Gras Lipides 26: 44. https://doi.org/ $10.1051 /$ ocl $/ 2019038$.

Duru M, Magrini MB. 2016. Consommer des produits dont les animaux ont été alimentés à l'herbe est-il suffisant pour équilibrer notre alimentation en acides gras poly-insaturés? Fourrages 228: 301-312.

Duru M, Therond O. 2019. «La «santé unique» pour reconnecter agriculture, environnement et alimentation ». Revue Regards RO8.

Duru M, Benoit M, Donnars C, Ryschawy J. 2017a. Quelle place pour l'élevage, les prairies et les produits animaux dans les transitions agricoles et alimentaires? Fourrages 232: 281-296.

Duru M, Bastien D, Froimont E, Graulet B. 2017b. Importance des produits issus de bovins au pâturage sur les apports nutritionnels et la santé du consommateur. Fourrages 230: 131-140.

Ecocert. 2021. Ecocert en cuisine. https://www.ecocert.com/fr-FR/ certification/referentiel-en-cuisine.

Ertl P, Knaus W, Zollitsch W. 2016. An approach to including protein quality when assessing the net contribution of livestock to human food supply. Animal 10(11): 1883-1889. https://doi.org/10.1017/ S1751731116000902. 
Fresán U, Sabaté J. 2019. Vegetarian diets: planetary health and its alignment with human health. Advances in Nutrition 10(Supplement_4): S380-S388. https://doi.org/10.1093/advances/ nmz019.

Gaudaré U, Pellerin S, Benoit M, Durand G, Dumont B, Barbieri P, et al. 2021. Comparing productivity and feed-use efficiency between organic and conventional livestock animals. Environmental Research Letters 16(2): 13. https://doi.org/10.1088/1748-9326/ abd65e.

Godfray HCJ, Aveyard P, Garnett T, Hall JW, Key TJ, Lorimer J, et al. 2018. Meat consumption, health, and the environment. Science (New York, N.Y.) 361(6399): 10. https://doi.org/10.1126/science. aam5324.

Herrero M, Henderson B, Havlik P, Thornton PK, Conant RT, Smith P, et al. 2016. Greenhouse gas mitigation potentials in the livestock sector. Nature Climate Change 6: 452-461. https://www.anses.fr/ fr/system/files/PASER-Sy-INCA2EN.pdf.

Kesse-Guyot E, Rebouillat P, Brunin J, Langevin B, Allès B, Touvier M, et al. 2021. Environmental and nutritional analysis of the EATLancet diet at the individual level: insights from the NutriNet-Santé study. Journal of Cleaner Production 296: 126555. https://doi.org/ 10.1016/j.jclepro.2021.126555.

Kim BF, Santo RE, Scatterday AP, Fry JP, Synk CM, Cebron SR, et al. 2020. Country-specific dietary shifts to mitigate climate and water crises. Global Environmental Change 101926. https://doi.org/ 10.1016/j.gloenvcha.2019.05.010.

Koch BJ, Hungate BA, Price LB. 2017. Food-animal production and the spread of antibiotic resistance: the role of ecology. Frontiers in Ecology and the Environment 15(6): 309-318. https://doi.org/ $10.1002 /$ fee. 1505 .

Kraimi N, Dawkins M, Gebhardt-Henrich SG, Velge P, Rychlik I, Volf $\mathrm{J}$, et al. 2019. Influence of the microbiota-gut-brain axis on behavior and welfare in farm animals: A review. Physiology and Behavior 210: 112658. https://doi.org/10.1016/j.phys beh.2019.112658.

Laboubee C, Couturier C, Bonhomme S, Damiano A, Hruschka S, Tignon E, et al. 2020. Methalae: Comment la méthanisation peut être un levier pour l'agroécologie? Innovations Agronomiques 79: 373-390. https://doi.org/10.15454/2zjv-rt73.

Lacour C, Seconda L, Allès B, Hercberg S, Langevin B, Pointereau P, et al. 2018. Environmental impacts of plant-based diets: how does organic food consumption contribute to environmental sustainability? Frontiers in Nutrition 5: 1-13. https://doi.org/10.3389/ fnut.2018.00008.

Laisse S, Baumont R, Dusart L, Gaudré D, Rouillé B, Benoit M, et al. 2019. L'efficience nette de conversion des aliments par les animaux d'élevage: une nouvelle approche pour évaluer la contribution de l'élevage à l'alimentation humaine. INRA Productions Animales 31 (3): 269-288. https://doi.org/10.20870/productions-anima les.2018.31.3.2355.

Lassaletta L, Billen G, Grizzetti B, Garnier J, Leach AM, Galloway JN. 2013. Food and feed trade as a driver in the global nitrogen cycle: 50-year trends. Biogeochemistry 118(1-3): 225-241. https:// doi.org/10.1007/s10533-013-9923-4.

Martin G, Durand JL, Duru M, Gastal F, Julier B, Litrico I, et al. 2020. Role of ley pastures in tomorrow's cropping systems. A review. Agronomy for Sustainable Development 40(3): 25. https://doi.org/ 10.1007/s13593-020-00620-9.

Michaud A, Plantureux S, Baumont R, Delaby L. 2021. Les prairies, une richesse et un support d'innovation pour des élevages de ruminants plus durables et acceptables. INRAE Productions Animales 33(3): 153-172. https://doi.org/10.20870/productionsanimales.2020.33.3.4543.
Miller GD, Comerford KB, Brown K, Mitloehner FM. 2020. The importance of a holistic approach to sustainability in dietary guidance. Journal of Nutrition Education and Behavior 52(2): 203-205. https://doi.org/10.1016/j.jneb.2019.10.019.

Ministère de la transition écologique et solidaire. 2020. Stratégie nationale bas carbone. https://www.ecologique-solidaire.gouv.fr/ sites/default/files/2020-03-25_MTES_SNBC2.pdf.

Mora O, Le Mouël C, de Lattre-Gasquet M, Donnars C, Dumas P, Réchauchère O, et al. 2020. Exploring the future of land use and food security: A new set of global scenarios. PloS One 15(7): e0235597. https://doi.org/10.1371/journal.pone.0235597.

Moraine M, Duru M, Therond O. 2016. A social-ecological framework for analyzing and designing integrated crop-livestock systems from farm to territory levels. Renewable Agriculture and Food Systems 32: 1-14. https://doi.org/10.1017/ S1742170515000526.

Mottet A, Haan C de, Falcucci A, Tempio G, Opio C, Gerber P. 2017. Livestock: On our plates or eating at our table? A new analysis of the feed/food debate. Global Food Security 14: 1-8. https://doi.org/ 10.1016/j.gfs.2017.01.001.

Mozaffarian D. 2020. Dietary and policy priorities to reduce the global crises of obesity and diabetes. Nature Food 1: 38-50. https:// doi.org/10.1038/s43016-019-0013-1.

Napoléone M, Gravas O, Rouquette A, Cittadini R, Campoy E. 2019. L'élevage et les friches au cœur de complémentarités entre littoral périurbain et arrière-pays. L'exemple du projet Fricato en Pyrénées Orientales. Innovations Agronomiques, Les polycultures-élevages : valoriser leurs atouts pour la transition agro-écologique 72: 107 119. https://doi.org/10.15454/vc2tz1.

Oliver TH, Boyd E, Balcombe K, Benton TG, Bullock JM, Donovan D, et al. 2018. Overcoming undesirable resilience in the global food system. Global Sustainability 1: 1-9. https://doi.org/10.1017/sus.2018.

Oliveira G de LT, Schneider M. 2015. The politics of flexing soybeans: China, Brazil and global agroindustrial restructuring. The Journal of Peasant Studies 43(1): 167-194. https://doi.org/ 10.1080/03066150.2014.993625.

Parlement européen. 2018. On a European strategy for the promotion of protein crops --encouraging the production of protein and leguminous plants in the European agriculture sector. https://www. europarl.europa.eu/doceo/document/A-8-2018-0121_EN.html.

Pellerin S, Bamière L, Angers D, Béline $\mathrm{F}$, Benoit $\mathrm{M}$, Butault $\mathrm{J}$, et al. 2013. Quelle contribution de l'agriculture française à la réduction des émissions de gaz à effet de serre ? Potentiel d'atténuation et coût de dix actions techniques. Rapport d'étude, INRA (France), 72 p.

Pellerin S, Bamière L, (pilotes scientifiques). 2019. Stocker du carbone dans les sols français. Quel potentiel au regard de l'objectif 4 pour 1000 et à quel coût? Synthèse du rapport d'étude. INRA (France), $114 \mathrm{p}$.

Perignon M, Vieux F, Soler LG, Masset G, Darmon N. 2017. Improving diet sustainability through evolution of food choices: Review of epidemiological studies on the environmental impact of diets. Nutrition Reviews 75(1): 2-17. https://doi.org/10.1093/nutrit/nuw043.

Pointereau P, Baudry J, Couturier C, Kesse-Guyot E, Hamon X, Hercberg S, et al. 2019. Le revers de notre assiette: changer d'alimentation pour préserver notre santé et notre environnement. Les résultats de l'étude BioNutrinet, $63 \mathrm{p}$.

Poore J, Nemecek T. 2018. Reducing food's environmental impacts through producers and consumers. Science 992(6392): 987-992. https://doi.org/10.1126/science.aaq0216.

Rémond D. 2016. Protéines animales-protéines végétales: Quel équilibre pour une alimentation saine et durable? In: Journées nationales des groupements techniques vétérinaires (JNGTV), Communication, 18-20 mai 2016, Nantes (France). 
Rinninella E, Cintoni M, Raoul P, Lopetuso LR, Scaldaferri F, Pulcini $\mathrm{G}$, et al. 2019. Health Gut Microbiota. Nutrients 1-23.

Robbins JA, Von Keyserlingk MAG, Fraser D, Weary DM. 2016. Invited review: Farm size and animal welfare. Journal of Animal Science 94(12): 5439-5455. https://doi.org/10.2527/jas.2016-0805.

Schader C, Muller A, Scialabba NEH, Hecht J, Isensee A, Erb KH, et al. 2015. Impacts of feeding less food-competing feedstuffs to livestock on global food system sustainability. Journal of The Royal Society Interface 12(113): 20150891. https://doi.org/ 10.1098/rsif.2015.0891.

Sanchez-Sabate R, Badilla-Briones Y, Sabaté J. 2019. Understanding attitudes towards reducing meat consumption for environmental reasons. A qualitative synthesis review. Sustainability 11: 6295. https://doi.org/10.3390/su11226295.

Santé publique France. 2018. Consommation d'antibiotiques et résistance aux antibiotiques en France. https://www.santepublique france.fr/les-actualites/2019/consommation-d-antibiotiques-et-anti bioresistance-en-france-en-2018.

Santo RE, Kim BF, Goldman SE, Dutkiewicz J, Biehl E, Bloem MW, et al. 2020. Considering plant-based meat substitutes and cellbased meats: A public health and food systems perspective. Frontiers in Sustainable Food Systems 4: 134. https://doi.org/ 10.3389 /fsufs. 2020.00134 .

Shi Z. 2019. Gut microbiota: An important link between western diet and chronic diseases. Nutrients 2287. https://doi.org/10.3390/ nu11102287.

Simopoulos AP, Di Nicolantonio JJ. 2016. The importance of a balanced $\omega-6$ to $\omega-3$ ratio in the prevention and management of obesity. Open Heart 3(2). https://doi.org/10.1136/openhrt-2015000385.

Sirami C, Gross N, Baillod AB, Bertrand C, Carrié R, Hass A, et al. 2019. Increasing crop heterogeneity enhances multitrophic diversity across agricultural regions. Proceedings of the National Academy of Sciences 116(33): 16442-16447. https://doi.org/ 10.1073/pnas.1906419116.

Smith P. 2014. Do grasslands act as a perpetual sink for carbon? Global Change Biology 20(9): 2708-2711. https://doi.org/10.1111/ gcb. 12561.

Smith LG, Kirk GJ, Jones PJ, Williams AG. 2019. The greenhouse gas impacts of converting food production in England and Wales to organic methods. Nature Communications 10(1): 1-10. https://doi. org/10.1038/s41467-019-12622-7.

Solagro. 2014. Afterres 2050. Un scénario soutenable pour l'agriculture et l'utilisation des terres en France à l'horizon 2050. Toulouse (France) : Éd. Solagro, 63 p.

Springmann M, Spajic L, Clark MA, Poore J, Herforth A, Webb P, et al. 2020. The healthiness and sustainability of national and global food-based dietary guidelines: a modelling study. The BMJ 1-16. https://doi.org/10.1136/bmj.m2322.

Stinner PW, Deuker A, Schmalfuß T, Brock C, Rensberg N, Denysenko V, et al. 2018. Legumes for soil health and sustainable management. Legumes for Soil Health and Sustainable Management. https://doi.org/10.1007/978-981-13-0253-4.

Temple D, Manteca X. 2020. Animal welfare in extensive production systems is still an area of concern. Frontiers in Sustainable Food Systems 4: 154. https://doi.org/10.3389/fsufs.2020.545902.

Thévenot A, Rivera JL, Wilfart A, Maillard F, Hassouna M, SengaKiesse T, et al. 2018. Mealworm meal for animal feed:
Environmental assessment and sensitivity analysis to guide future prospects. Journal of Cleaner Production 170: 1260-1267. https:// doi.org/10.1016/j.jclepro.2017.09.054.

Thorlakson T, De Zegher JF, Lambin EF. 2018. Companies' contribution to sustainability through global supply chains. Proceedings of the National Academy of Sciences of the United States of America 115(9): 2072-2077. https://doi.org/10.1073/ pnas. 1716695115.

Van Kernebeek HRJ, Oosting SJ, Van Ittersum MK, Bikker P, De Boer IJM. 2016. Saving land to feed a growing population: consequences for consumption of crop and livestock products. International Journal of Life Cycle Assessment 21(5): 677-687. https://doi.org/10.1007/s11367-015-0923-6.

Van Wagenberg CPA, De Haas Y, Hogeveen H, Van Krimpen MM, Meuwissen MPM, Van Middelaar CE, et al. 2017. Animal Board Invited Review: Comparing conventional and organic livestock production systems on different aspects of sustainability. Animal 11(10): 1839-1851. https://doi.org/10.1017/ S175173111700115X.

Varijakshapanicker P, Mckune S, Miller L, Hendrickx S, Dahl GE, Adesogan AT. 2019. Sustainable livestock systems to improve human health, nutrition, and economic status. Animal Frontiers 9 (4). https://doi.org/10.1093/af/vfz041.

Vazeille K, Veysset P, Note P, Sepchat B, Salle G, Dhour P, et al. 2018. Interdisciplinary design of low-input grass-fed livestock systems: the example of the Salamix system experiment. In: $24^{e}$ Rencontres autour des Recherches sur les Ruminants (3R), Institut de l'Elevage-INRA, France.

Vieux F, Darmon N, Touazi D, Soler LG. 2012. Greenhouse gas emissions of self-selected individual diets in France: Changing the diet structure or consuming less? Ecological Economics 75: $91-$ 101. https://doi.org/10.1016/j.ecolecon.2012.01.003.

Weindl I, Ost M, Wiedmer P, Schreiner M, Neugart S, Klopsch R, et al. 2020. Sustainable food protein supply reconciling human and ecosystem health: A Leibniz Consensus. Global Food Security 25: 100367. https://doi.org/10.1016/j.gfs.2020.100367.

Weishaupt A, Ekardt F, Garske B, Stubenrauch J, Wieding J. 2020. Land use, livestock, quantity governance, and economic instruments-sustainability beyond big livestock herds and fossil fuels. Sustainability 12(5). https://doi.org/10.3390/su12052053.

Westhoek H, Lesschen JP, Rood T, Wagner S, De Marco A, MurphyBokern D, et al. 2014. Food choices, health and environment: Effects of cutting Europe's meat and dairy intake. Global Environmental Change 26: 196-205. https://doi.org/10.1016/j. gloenvcha.2014.02.004.

Wilkinson DA, Marshall JC, French NP, Hayman DTS. 2018. Habitat fragmentation, biodiversity loss and the risk of novel infectious disease emergence. Journal of the Royal Society Interface 15(149): 10. https://doi.org/10.1098/rsif.2018.0403.

Willett W, Rockström J, Loken B, Springmann M, Lang T, Vermeulen S, et al. 2019. Food in the Anthropocene: the EAT-Lancet Commission on healthy diets from sustainable food systems. Lancet 6736(18): 3-49. https://doi.org/10.1016/S0140-6736(18) 31788-4.

Zu Ermgassen EKHJ, Phalan B, Green RE, Balmford A. 2016. Reducing the land use of EU pork production: Where there's swill, there's a way. Food Policy 58: 35-48. https://doi.org/10.1016/j.foodpol.2015.11.001.

Citation de l'article : Duru M, Le Bras C, Grillot M. 2021. Une approche holistique de l'élevage, au cœur des enjeux de santé animale, humaine et environnementale. Cah. Agric. 30: 26. 